\title{
INFLUÊNCIA DA ADUBAÇÃO ORGÂNICA NO CRESCIMENTO DE TANGERINEIRA cv Clemenules E NOS ATRIBUTOS QUÍMICOS E MICROBIOLÓGICOS DO SOLO ${ }^{1}$
}

\author{
GIOVAM BATTISTA SORRENTI ${ }^{2}$, JOSÉ CARLOS FACHINELLO ${ }^{3}$, DANILO DUFECHCASTILHOS ${ }^{3}$, \\ VALMOR JOÃO BIANCHI ${ }^{4}$, BRUNO MARANGONI $^{2}$
}

RESUMO - O estudo objetivou avaliar o efeito de adubos orgânicos de diferentes origens, empregados como fontes de nitrogênio, sobre a atividade vegetativa das plantas de tangerineira (cv Clemenules/Poncirus trifoliata) durante a fase inicial de desenvolvimento e atributos químicos e microbiológicos do solo, nas condições pedológicas e climáticas da região de Pelotas-RS. O experimento foi conduzido durante dois anos (2002-2003), utilizando-se de plantas de 18 meses de idade, em vasos de $35 \mathrm{~kg}$ de capacidade, irrigadas por gotejamento, com seis repetições, sendo cada unidade experimental constituída por uma planta. Os tratamentos confrontados foram os seguintes: Controle (sem adubação); Adubação mineral (AM); Vermicomposto (VC); Vermicomposto + sangue bovino seco $(\mathrm{VC}+\mathrm{SB})$ e Resíduo da indústria de transformação de sucos cítricos (RITC). Ao final de cada estação vegetativa, foram avaliados os seguintes parâmetros biométricos: área de seção do porta-enxerto, altura da copa, número de brotações, folhas totais produzidas por planta e massa fresca e seca das folhas. Ao fim do experimento, três plantas de cada tratamento foram desplantadas, separados os principais órgãos, determinando-se a área total das folhas produzidas, a massa fresca e seca dos ramos principal e secundários e das raízes, separadas em grossas e finas. Na mesma época, amostras de solo de cada vaso foram coletadas e utilizadas para as análises químicas e microbiológicas. Em ambos os anos, foram coletadas folhas avaliando-se a concentração dos macronutrientes. Os efeitos mais marcantes sobre o desenvolvimento das plantas foram observados naquelas em que, além do vermicomposto (VC), foi fornecido periodicamente sangue seco $(\mathrm{VC}+\mathrm{SB})$. No solo desses tratamentos, também foi observado aumento do teor de nitrogênio total e da atividade microbiana do solo, avaliada pela respiração basal. $\mathrm{O}$ aporte de sangue seco $(\mathrm{VC}+\mathrm{SB})$ supriu satisfatoriamente as necessidades de $\mathrm{N}$ ocorrido nas plantas fertilizadas com adubo orgânico mesmo que estas últimas tenham recebido, no biênio de experimentação, cerca de $40 \%$ a menos de nitrogênio em relação às plantas adubadas com a fonte mineral (AM). Por isso, os tratamentos VC e RITC induziram um efeito limitado no desenvolvimento das plantas, enquanto, provavelmente, estas duas últimas fontes, e nas quantidades utilizadas, não forneceram suficientemente nitrogênio disponível para suprir a demanda das plantas. De maneira geral, a concentração dos macronutrientes foliares foi adequada em relação ao $\mathrm{P}, \mathrm{K}, \mathrm{Ca}$ e $\mathrm{Mg}$ e abaixo do normal para o $\mathrm{N}$, demonstrando a elevada exigência desse nutriente na fase inicial de crescimento e desenvolvimento de tangerineiras cv. Clemenules. Termos para indexação: Adubo orgânico, nitrogenio, vermicomposto, sangue bovino seco, respiração do solo.

\section{EFFECT OF DIFFERENT ORGANIC FERTILIZATION STRATEGIES ON VEGETATIVE GROWTH AND SOIL PROPERTIES OF NON BEARING CITRUS TREES (cv Clemenules)}

ABSTRACT - In order to evaluate the effect of different organic fertilization strategies, as nitrogen source, on vegetative growth of young citrus plants as well as on chemical and microbiological soil properties, a two-year experiment was performed on eighteen months old citrus plants (cv Clemenules) grafted on Poncirus trifoliata. The trees were transplanted in $35 \mathrm{~L}$ pots filled with a sandyloam soil and drip irrigation. The following treatments were compared: Control (unfertilized); Mineral fertilizer (MF); Vermicompost (VC); Vermicompost + blood meal $(\mathrm{VC}+\mathrm{BM})$; Citrus compost (CC). Each treatment was applied to 6 plants (replicates). At the end of each vegetative season the following parameters were measured: rootstock section area, plant height, leaf and shoot number per plant, leaf fresh and dry matter. Three plants per treatment were harvested and leaf total area, branches and roots (divided in fine and coarse) fresh and dry matter were recorded. In addition, soil samples of each pot were collected and soil chemical and microbiological properties were measured. Plant growth was positively affected by VC $+\mathrm{BM}$ fertilization strategy in comparison to the other treatments. Such effect was observed despite the total amount of $\mathrm{N}$ supplied to VC $+\mathrm{BM}$, was about $40 \%$ lower than the $\mathrm{N}$ applied to plants by mineral source MF. The supply of blood meal contributed to avoid the temporary soil N-deficiency occurred when organic fertilizer is incorporated into the soil. VC and CC strategies induced limited effects on plant growth likely due to the lower soil $\mathrm{N}$-availability than the plant requirements. Moreover, only $\mathrm{VC}+\mathrm{SB}$ promoted soil total $\mathrm{N}$ and microbial soil respiration. Generally, the macronutrient concentration in the leaves was adequate in relation to $\mathrm{P}, \mathrm{K}, \mathrm{Ca}$ and $\mathrm{Mg}$, except for $\mathrm{N}$, giving evidence of the high request for $\mathrm{N}$ at the beginning of growing and development stage of Clemenules tangerines.

Index Terms: Organic fertilizers, nitrogen, vermicompost, blood meal, soil respiration.

'(Trabalho 005-08). Recebido em: 02-01-2008. Aceito para publicação em: 09-09-2008.

${ }^{2}$ Dep. to de Colture Arboree, Università di Bologna, Viale G. Fanin 46, 40128 Bologna, Italia: sorrenti@agrsci.unibo.it

${ }^{3}$ Faculdade de Agronomia Eliseu Maciel, C. Postal 354, 9600701 Pelotas - RS, Brasil: jfachi@ufpel.tche.br

${ }^{4}$ Instituto de Biologia da Ufpel, C. Postal 354, 9600701 Pelotas - RS, Brasil

Rev. Bras. Frutic., Jaboticabal - SP, v. 30, n. 4, p. 1129-1135, Dezembro 2008 


\section{INTRODUÇÃO}

O Brasil é um tradicional produtor de frutas cítricas, liderando a produção mundial e a exportação de suco cítrico concentrado (FAO, 2006). São Paulo é o maior Estado produtor, porém o Rio Grande do Sul é citado como o Estado que possui as melhores condições climáticas para produzir frutas cítricas de alta qualidade, principalmente para consumo in natura. Contudo, a produção orgânica de laranjas representa um percentual pequeno do setor agrícola brasileiro, sendo realizada numa superfície de somente alguns hectares, mesmo considerando as enormes potencialidades que o País apresenta.

A nutrição representa uma das técnicas agronômicas capazes de influenciar nos rendimentos quali-quantitativos das produções frutícolas. Todavia, conjugar as exigências produtivas com aquelas ecológicas representa, atualmente, um binômio imprescindível (Sorrenti, 2006). Embora a nutrição mineral seja de mais fácil aplicação e de menor custo, a adoção da fertilização orgânica melhora as propriedades físicas, químicas e microbiológicas do solo, gerando um menor impacto ambiental (Peducci, 1992; Giusquiniani et al., 1995; Jorgensen et al., 1996; Paroda, 1999; Dahama, 2002). A adoção de estratégias e fontes alternativas de nutrientes é importante para manter bons índices produtivos e a qualidade da fruta cítrica, principalmente na produção orgânica (Huchche et al., 1998), cujo produto possui maior valor no mercado (French, 1990).

Dentre as estratégias de fertilização de plantas frutíferas, é notório como o nitrogênio se torna o principal fator limitante no cultivo orgânico (Scudellari et al., 1998). Além disso, a utilização de adubo elaborado a partir de resíduos orgânicos descartados pelas indústrias e, até mesmo, o lixo das cidades oferecem uma ótima perspectiva de reciclagem de compostos orgânicos (Toselli et al., 2004).

Embora exista uma recomendação geral sobre o uso de adubos orgânicos em plantas cítricas, dados científicos comparativos ainda são incipientes. Estudos realizados por Almeida et al. (2005), sobre o efeito de esterco de curral curtido na adubação de formação do pomar de tangerineira 'Poncã' (Citrus reticulata, Blanco), em doses crescentes $(0,4 ; 7,5 ; 15,0$; 22,5 e 30,0 kg planta-1), comparando com a adubação química convencional, concluíram que, na média das avaliações, houve aumento linear das características de crescimento das plantas com o incremento das doses de esterco de curral curtido, e que não houve diferença significativa entre os tratamentos com adubo orgânico e químico.

O estudo objetivou avaliar o efeito de adubos orgânicos de diferentes origens, empregados como fontes de nitrogênio, sobre a atividade vegetativa das plantas de tangerineira $\mathrm{cv}$ Clemenules durante a fase inicial de desenvolvimento e os atributos químicos e microbiológicos do solo, nas condições pedológicas e climáticas da região de Pelotas -RS.

\section{MATERIAL E MÉTODOS}

O experimento, com duração de dois anos, foi conduzido na área experimental do Departamento de Fitotecnia da Faculdade de Agronomia "Eliseu Maciel" (FAEM) da Universidade Federal de Pelotas (UFPel), Pelotas-RS, tendo início no mês de novembro de 2001. Utilizaram-se plantas de tangerineira cv Clemenules enxertadas sobre Poncirus trifoliata, com 18 meses de idade. Por ocasião do transplantio, as plantas apresentavam-se em haste única de altura média de $64,3 \pm 6,4 \mathrm{~cm}$ a partir do colo, com 12,5 \pm 4,9 folhas completamente expandidas e com 42,1 $\pm 9,7 \mathrm{~mm}^{2}$ de área da seção do caule. As plantas foram acondicionadas em vasos, utilizando como substrato a camada superficial $(0-10 \mathrm{~cm})$ de um Argissolo Vermelho- Amarelo (35 kg planta ${ }^{-1}$ ), cujas características principais, baseadas na análise laboratorial, estão descritas na Tabela 1. Antes do plantio, misturou-se o calcário $\left(330 \mathrm{~g} \mathrm{~m}^{-3}\right)$ com PRNT de $80 \%$ no solo para elevar o $\mathrm{pH}$ a 6,0. A irrigação das plantas foi feita por gotejamento, e o conteúdo de água nos vasos foi controlado com o auxílio de dois tensiômetros, a fim de manter o solo com capacidade hídrica de campo.

Para o experimento, foram testadas diversas fontes de nitrogênio: uma fonte mineral (AM) e diferentes fontes orgânicas, entre as quais: vermicomposto (VC) (obtido mediante mistura de esterco bovino, capim e folhas de plantas em compostagem com minhocas), sangue bovino seco (SB) $(12,9 \% \mathrm{~N})$ e resíduo da indústria de transformação de sucos cítricos (RITC), previamente compostado e misturados em todo o solo. As principais caraterísticas fisico-químicas do vermicomposto e do RITC utilizados no experimento estão resumidas na Tabela 2.

Os tratamentos avaliados foram os seguintes: Controle (sem adubação); Adubo mineral (AM); Vermicomposto (VC); Vermicomposto + sangue bovino seco $(\mathrm{VC}+\mathrm{SB})$; e Resíduo da indústria de transformação de sucos cítricos (RITC).

As doses e as épocas de fertilização do solo, nos dois anos de experimentação, estão resumidas na Tabela 3.

Ao final de cada estação vegetativa, foram avaliados os seguintes parâmetros biométricos: área de seção do porta-enxerto $\left(\mathrm{mm}^{2}\right)$ medido a $10 \mathrm{~cm}$ abaixo do ponto de enxertia; altura máxima da copa $(\mathrm{cm})$ a partir do colo, número de brotações, folhas totais produzidas por plantas e a massa fresca e seca das folhas (g). Em ambos os anos, de todas as plantas, foram coletadas folhas da porção mediana do ramo da estação de crescimento, avaliandose a concentração dos macronutrientes, N, P, K, Ca e Mg. Ao fim do experimento, em julho de 2003, três plantas de cada tratamento foram desplantadas, separados os principais órgãos, determinando-se a área total das folhas produzidas $\left(\mathrm{cm}^{2}\right)$, a massa fresca e seca dos ramos principal e secundários (g) e das raízes (g), separadas em grossas e finas. Além disso, foram coletadas amostras de $450 \mathrm{~g}$ de solo de cada tratamento (amostras compostas obtidas de dois pontos de coleta por vaso, de todo o experimento) com auxílio de um trado até o fundo dos vasos das quais foram determinados os teores de carbono orgânico e de nitrogênio total, conforme metodologias descritas em Tedesco et al. (1995). A biomassa microbiana foi determinada pelo método descrito por Vance et al. (1987), utilizando-se, em substituição ao clorofórmio, do forno de microondas, freqüência de $2.450 \mathrm{MHz}$ e energia de $1,35 \mathrm{KW}$, por qutro minutos, provocando a liberação dos componentes celulares para a eliminação dos microrganismos, conforme sugerido por Ferreira et al. (1999). A 
respiração basal do solo foi determinada pela quantificação do dióxido de carbono $\left(\mathrm{CO}_{2}\right)$ liberado no processo de respiração microbiana, a partir de $100 \mathrm{~g}$ de solo, durante 21 dias de incubação, conforme metodologia de Stotzky (1965).

$\mathrm{O}$ experimento foi disposto em delineamento em blocos ao acaso, com seis repetições, sendo cada unidade experimental constituída por uma planta, totalizando 30 plantas. Os dados obtidos foram submetidos à análise da variância, utilizando-se do procedimento GLM do software SAS (SAS Institute Cary, NC, USA). A separação das médias foi obtida com o teste de Student $(\mathrm{P} \leq 0,05)$.

\section{RESULTADOS E DISCUSSÃO}

As diferentes estratégias de fertilização proporcionaram efeitos significativos sobre as características biométricas de tanjerineira já no primeiro ano de experimentação e sobre os atributos químicos e microbiológicos do solo verificados ao final do experimento.

As avaliações feitas ao final da primeira estação vegetativa revelaram que o tratamento $\mathrm{VC}+\mathrm{SB}$ determinou um incremento da área de seção da haste principal, porém nenhum dos tratamentos influenciaram de maneira significativa a altura total e o número de brotações por planta (Tabela 4). Na mesma época, evidenciou-se, em todos os tratamentos, maior número de folhas por planta quando comparado ao controle, registrandose valores mais elevados no tratamento $\mathrm{VC}+\mathrm{SB}$. De maneira geral, apesar de ter ocorrido aumento da percentagem da massa seca das folhas em todos os tratamentos, somente o uso de composto cítrico induziu um incremento significativo da massa fresca e seca foliar, comparando-se ao controle (Tabela. 5).

No segundo ciclo vegetativo, as diferenças no desenvolvimento das plantas entre os tratamentos área do tronco e matéria seca dos tecidos analisados foram mais acentuadas, sendo particularmente estimuladas pelo tratamento $\mathrm{VC}+\mathrm{SB}$, seguido pelo AM, em todas as variáveis analisadas (Tabelas 4, 5 e 6).

Durante a fase de crescimento, a quantidade dos elementos minerais absorvida pelas plantas é determinada pelo desenvolvimento das porções perenes e estruturais (Toselli et al., 2004). Na prática, quando é feita adequada adubação de base, o desenvolvimento das plantas jovens, durante a fase de condução, pode ser obtido com o manejo adequado da água e do nitrogênio. Os efeitos mais marcantes sobre o desenvolvimento das plantas, tanto no primeiro quanto no segundo ciclo vegetativo, foram observados nas plantas em que, além do vermicomposto, foi fornecido periodicamente sangue seco, mesmo que estas últimas tenham recebido, no biênio de experimentação, cerca de $40 \%$ a menos de nitrogênio em relação às plantas adubadas com a fonte de nitrogênio mineral (AM). Devido à ótima disponibilidade de nitrogênio orgânico (12,9\%), presente numa forma facilmente mineralizável (proteínas do sangue), o aporte de sangue seco supriu satisfatoriamente o estado de carência temporária de $\mathrm{N}$ ocorrido nas plantas fertilizadas com adubo orgânico. A carência temporária foi caracterizada pela diminuição do crescimento das plantas. O N está imobilizado no adubo orgânico e será mineralizado. Nos adubos minerais, o $\mathrm{N}$ já está na forma disponível. Quando se realiza a fertilização exclusiva com adubos orgânicos, pode determinar, na fase inicial, uma parcial imobilização de nitrogênio no solo devido ao aumento da demanda por parte da microflora e microfauna do solo, determinando uma carência temporária desse elemento para as plantas (Mazzanti, 2002).

Diante disso, verificou-se que o aporte ao solo dos compostos orgânicos nos tratamentos VC e RITC induziu um efeito limitado no desenvolvimento das plantas. Provavelmente, estas duas últimas fontes, e nas quantidades utilizadas, não forneceram suficientemente nitrogênio disponível para suprir a demanda das plantas. Além disso, o nitrogênio fornecido na forma orgânica pelo sangue seco, devido à rápida mineralização, contribui para maior eficiência, sendo que a matéria orgânica no solo contribuiu para limitar as perdas de $\mathrm{N}$ por lixiviação, conforme evidenciado pelo conteúdo de $\mathrm{N}$ total no solo ao final do experimento. $\mathrm{Na}$ adubação convencional, em que o aporte de nitrogênio ao solo é feito na forma mineral, pode haver perdas por lixiviação; porém, na adubação orgânica, as perdas são menores, logo após as freqüentes e abundantes precipitações, típicas das regiões meridionais do Brasil durante os períodos de primavera e verão (que podem chegar a $2.000 \mathrm{~mm} \mathrm{ano}^{-1}$ ), contribuindo para afastar as frações de nitrogênio, muito móveis $\left(\mathrm{NO}_{3}^{-}\right)$(Scudellari et al., 1998).

A concentração dos macronutrientes foliares na tanjerineira Clemenules foi influenciada pelos tratamentos nos dois anos do ensaio (Tabela 7). Embora a concentração de nitrogênio tenha aumentado em VC $+\mathrm{SB}$ e RITC nas duas estações vegetativas e em AM, no segundo ano, de maneira geral, o teor de $\mathrm{N}$ foliar registrado em todos os tratamentos deve ser considerado abaixo do nível-padrão para a espécie, pois o nível normal está na faixa de 2,3-2,7 (Comissão de Fertilidade do Solo-RS/SC, 2004) e, particularmente em VC, demonstrando a elevada exigência de nitrogênio na fase inicial de crescimento e desenvolvimento das plantas jovens (Scudellari et al., 1998). A concentração de $\mathrm{P}, \mathrm{Ca}$ e $\mathrm{Mg}$ nas folhas, em ambos os anos de avaliação, e de $\mathrm{K}$, no primeiro ano, revelou-se dentro dos níveis mínimos exigidos pela cultura (Jones et al., 1991; Malavolta et al., 1996; Calabrese et al., 1998), mesmo havendo diferenças, em todos os tratamentos. No segundo ano de cultivo, verificou-se que a concentração de fósforo nas folhas aumentou somente nas plantas que receberam aporte de matéria orgânica ao solo. É notório como a disponibilidade de fósforo pelas plantas é sensivelmente incrementada pelo aporte de fertilizante orgânico, em particular nos solos com $\mathrm{pH}$ abaixo de 6, o qual pode estimular a estabilização das micorrizas (Schubert et al., 1992), associação mutualística entre a planta e os fungos, em que o porta-enxerto empregado no experimento (Poncirus trifoliata) é favorecido (Qiangsheng et al., 2006; Srivastava et al., 2002).

Ao final do ensaio, a concentração de $\mathrm{K}$ revelou-se significativamente reduzida nas folhas de tangerineira sob todos os tratamentos, quando comparada à testemunha, estabilizandose abaixo do nível crítico para a espécie (Jones et al., 1991), particularmente em RITC, evidenciando a necessidade de monitorar, além do nitrogênio, a disponibilidade no solo dos 
outros nutrientes durante a fase de crescimento das plantas.

As análises conduzidas para avaliar os efeitos das adubações sobre os atributos químicos e microbiológicos do solo, após dois anos de condicionamento do mesmo, revelaram efeitos significativos nas variáveis analisadas, com exceção do teor de nitrogênio microbiano (Tabela 8).

Entre as diferentes estratégias de fertilização, os tratamentos com vermicomposto e vermicomposto + sangue bovino aumentaram o teor de nitrogênio total, sendo que a atividade microbiana do solo, avaliada pela respiração basal, só foi influenciada de forma significativa pelo vermicomposto + sangue bovino. $\mathrm{O}$ mesmo tratamento apresentou maior valor de carbono microbiano, significativamente superior ao tratamento onde se aplicou composto de citros, mas igual aos demais tratamentos.

Numerosos estudos têm evidenciado como a atividade biológica do solo, devido à aplicação de matéria orgânica, está relacionada com o aumento da respiração do solo (Jorgensen et al., 1996; Niklasch \& Jorgensen, 2001).
No presente trabalho, verificou-se que o tratamento contendo vermicomposto + sangue apresentou valor de respiração basal significativamente maior $(45 \% \pm 5)$ que os demais tratamentos, o que tende a colaborar para melhor ambiente de desenvolvimento para as plantas. Esse efeito está, provavelmente, ligado à integração da matéria orgânica ao solo adicionada com sangue seco e vermicomposto, que, disponibilizando o aporte de nitrogênio, estimulou a atividade de proliferação microbiana no solo, acelerando os processos de degradação da substância orgânica (Tabela 8), tornando disponível maiores quantidades de $\mathrm{N}$ e outros nutrientes para as plantas (Stevenson, 1986). As análises microbiológicas conduzidas com o solo das plantas fertilizadas com substâncias orgânicas, nos tratamentos VC e RITC, não evidenciaram diferenças significativas em relação aos valores obtidos nas plantas-testemunha para todas as variáveis analisadas. $\mathrm{O}$ aporte de matéria orgânica no solo nem sempre implica, num curto período, uma resposta sobre a biomassa microbiana do solo, assim como observado em condições de campo sobre plantas adultas de pessegueiro (Baldi et al., 2006). Essa resposta também pode estar relacionada às características do substrato empregado.

TABELA 1- Principais características físico-químicas e microbiológicas do solo usado no experimento. FAEM/UFPEL.

\begin{tabular}{lc}
\hline Parâmetro/Método de análise do solo & Valor \\
\hline Peso específico $\left(\mathrm{t} \mathrm{m}^{-3}\right)$ & 1,35 \\
Argila (\%) & 18,0 \\
pH em $\mathrm{H}_{2} \mathrm{O}$ & 5,7 \\
Índice SMP & 6,5 \\
Matéria orgânica (Wakley-Black) $(\%)$ & 2,89 \\
Nitrogênio total $\left(\right.$ Kjeldahl) $\left(\mathrm{g} \mathrm{kg}^{-1}\right)$ & 1,23 \\
Nitrogênio microbiano $\left(\mu \mathrm{g} \mathrm{g}^{-1}\right)$ & 5,8 \\
Carbono orgânico $(\%)$ & 1,70 \\
Carbono microbiano $\left(\mu \mathrm{g} \mathrm{g}^{-1}\right)$ & 60,6 \\
$\mathrm{C} / \mathrm{N}$ & 13,8 \\
$\mathrm{P}\left(\right.$ Mehlich 1) $\left(\mathrm{mg} \mathrm{kg}^{-1}\right)$ & 19,3 \\
$\mathrm{~K}\left(\right.$ Mehlich 1) $\left(\mathrm{mg} \mathrm{kg}^{-1}\right)$ & 126 \\
$\mathrm{Na}\left(\right.$ Mehlich 1) $\left(\mathrm{mg} \mathrm{kg}^{-1}\right)$ & 3,0 \\
$\mathrm{Mg}($ extração $\mathrm{KCl})\left(\mathrm{cmol}_{\mathrm{c}} \mathrm{dm}^{-3}\right)$ & 2,2 \\
$\mathrm{Al}($ extração $\mathrm{KCl})\left(\mathrm{cmol}_{\mathrm{c}} \mathrm{dm}^{-3}\right)$ & 0,1 \\
$\mathrm{Ca}($ extração $\mathrm{KCl})\left(\mathrm{cmol}_{\mathrm{c}} \mathrm{dm}^{-3}\right)$ & 2,7 \\
$\mathrm{Respiração} \mathrm{Basal}\left(\mathrm{mg} \mathrm{CO}_{2}^{-1} 100 \mathrm{~g}^{-1}\right.$ solo) & 25,6 \\
\hline
\end{tabular}

TABELA 2- Principais características físico-químicas e microbiológicas do Vermicomposto (V), Resíduo da indústria de suco cítrico (RITC) e teor de $\mathrm{N}$ no sangue bovino usados no experimento. FAEM/UFPEL.

\begin{tabular}{|c|c|c|}
\hline Determinações & $(V)$ & (RITC) \\
\hline Umidade (\%) & 70,9 & 65,4 \\
\hline $\mathrm{pH}$ & 8,6 & 8,2 \\
\hline Densidade $\left(\mathrm{g} \mathrm{cm}^{-3}\right)$ & i & 0,8 \\
\hline C orgânico (Wakley-Black) (\%) & 20,5 & 32,0 \\
\hline Nitrogênio total (Kjeldahl) (\%) & 2,05 & 3,2 \\
\hline $\mathrm{C} / \mathrm{N}$ & 9,76 & 10,0 \\
\hline Fósforo total (\%) & 0,69 & 0,44 \\
\hline Potássio total (\%) & 0,34 & 0,24 \\
\hline Cálcio total (\%) & 3,0 & 7,6 \\
\hline Magnésio total (\%) & 0,16 & 0,2 \\
\hline Ferro total $(\%)$ & / & 1,6 \\
\hline Sódio total $(\%)$ & / & 0,34 \\
\hline Enxofre total (\%) & / & 0,64 \\
\hline Cobre total $\left(\mathrm{mg} \mathrm{kg}^{-1}\right)$ & / & 32,0 \\
\hline Zinco total $\left(\mathrm{mg} \mathrm{kg}^{-1}\right)$ & / & 62,0 \\
\hline Manganês total $\left(\mathrm{mg} \mathrm{kg}^{-1}\right)$ & / & 379,0 \\
\hline Sangue bovino $(\% \mathrm{~N})$ & 13,9 & \\
\hline
\end{tabular}


TABELA 3 - Doses e épocas de aplicação das diferentes fontes de nitrogênio utilizadas nos dois anos de experimentação, FAEM/ UFPEL.

\begin{tabular}{|c|c|c|c|c|c|c|}
\hline & \multicolumn{3}{|c|}{ Primeiro ano de cultivo } & \multicolumn{3}{|c|}{ Segundo ano de cultivo } \\
\hline Tratamento & $\begin{array}{c}\text { Dose } \\
\left(\mathrm{g} \mathrm{planta}^{-1}\right)\end{array}$ & $\begin{array}{c}\mathrm{N}^{2} \\
\left(\text { g planta }^{-1}\right)\end{array}$ & Época & $\begin{array}{c}\text { Dose } \\
\left(\mathrm{g} \mathrm{planta}^{-1}\right)\end{array}$ & $\begin{array}{c}\mathrm{N}^{2} \\
\left(\text { g planta }^{-1}\right)\end{array}$ & Época \\
\hline Controle & - & - & - & - & - & - \\
\hline Mineral & $\begin{array}{l}33 \\
20\end{array}$ & $\begin{array}{l}5 \\
3\end{array}$ & $\begin{array}{l}13-12-01 \\
35 \text { DAPA }^{4}\end{array}$ & $\begin{array}{r}59 \\
36 \\
24\end{array}$ & $\begin{array}{c}9 \\
5,4 \\
3,6 \\
\end{array}$ & $\begin{array}{l}20 \text { DAIB } \\
30 \text { DAPA } \\
30 \text { DASA }\end{array}$ \\
\hline Vermicomposto & 460 & 2,7 & Transplantio & 550 & & $30 \mathrm{DAB}$ \\
\hline $\begin{array}{l}\text { Vermicomposto } \\
+ \text { sangue }\end{array}$ & $\begin{array}{l}460(\mathrm{~V})^{3} \\
24(\mathrm{SB})^{3} \\
16(\mathrm{SB})\end{array}$ & $\begin{array}{l}2,7 \\
3,0 \\
2,0\end{array}$ & $\begin{array}{c}\text { Transplantio } \\
13-12-01 \\
35 \text { DAPA }\end{array}$ & $\begin{array}{l}550(\mathrm{~V}) \\
24(\mathrm{SB}) \\
16(\mathrm{SB}) \\
16(\mathrm{SB})\end{array}$ & $\begin{array}{l}3,2 \\
3,0 \\
2,0 \\
2,0\end{array}$ & $\begin{array}{l}30 \mathrm{DAB} \\
20 \mathrm{DAIB} \\
30 \mathrm{DAPA} \\
30 \text { DASA }\end{array}$ \\
\hline $\begin{array}{l}\text { Composto } \\
\text { cítrico }\end{array}$ & 460 & 5,1 & Transplantio & 550 & 6,0 & $30 \mathrm{DA} B$ \\
\hline
\end{tabular}

${ }^{1}$ Dose baseada na recomendação de adubação para citros no RS como adubo mineral.

${ }^{2}$ Como fonte de nitrogênio, utilizaram-se $\mathrm{Ca}\left(\mathrm{NO}_{3}\right)_{2}(15 \% \mathrm{~N}$ e $19 \% \mathrm{Ca})$ e Uréia $(45 \% \mathrm{~N})$ no primeiro e segundo anos de cultivo, respectivamente.

${ }^{3} \mathrm{~V}=$ Vermicomposto; $\mathrm{SB}=$ sangue bovino seco;

${ }^{4}$ DAPA = Dias após a primeira aplicação; DAIB= Dias após o início da brotação; DASA = Dias após a segunda aplicação; DAB= Dias antes da brotação.

TABELA 4- Médias das características biométricas das plantas no primeiro e no segundo anos, FAEM/UFPEL.

\begin{tabular}{|c|c|c|c|c|c|c|c|c|}
\hline \multirow[t]{2}{*}{ Tratamento $^{1}$} & \multicolumn{2}{|c|}{ Área do tronco $\left(\mathrm{mm}^{2}\right)$} & \multicolumn{2}{|c|}{ Altura da planta $(\mathrm{cm})$} & \multicolumn{2}{|c|}{$\begin{array}{l}\text { Número de brotações } \\
\text { plantas }^{-1}\end{array}$} & \multicolumn{2}{|c|}{ Número de folhas plantas } \\
\hline & $1^{\circ}$ ano & $2^{\circ}$ ano & $1^{\circ}$ ano & $2^{\circ}$ ano & $1^{\circ}$ ano & $2^{\circ}$ ano & $1^{\circ}$ ano & $2^{\circ}$ ano \\
\hline Controle & $104,6 \mathrm{~b}$ & $267,2 \mathrm{~d}$ & 89,8 & $97 \mathrm{~b}$ & 5,0 & $53 \mathrm{c}$ & $83 \mathrm{c}$ & $236 \mathrm{~b}$ \\
\hline AM & $111,3 \mathrm{~b}$ & $528,4 \mathrm{~b}$ & 88,5 & $109 \mathrm{ab}$ & 8,3 & $82 \mathrm{~b}$ & $154 \mathrm{~b}$ & $780 \mathrm{a}$ \\
\hline VC & $106,1 \mathrm{~b}$ & $383,5 \mathrm{c}$ & 84,8 & $96 \mathrm{~b}$ & 7,5 & $74 \mathrm{bc}$ & $160 \mathrm{~b}$ & $419 \mathrm{~b}$ \\
\hline $\mathrm{VC}+\mathrm{SB}$ & $150,0 \mathrm{a}$ & $635,2 \mathrm{a}$ & 100,8 & $115 \mathrm{a}$ & 10,0 & $112 \mathrm{a}$ & $237 \mathrm{a}$ & $855 \mathrm{a}$ \\
\hline RIC & $98,9 \mathrm{~b}$ & $348,2 \mathrm{~cd}$ & 86,7 & $98 \mathrm{~b}$ & 7,2 & $51 \mathrm{c}$ & $136 \mathrm{~b}$ & $313 \mathrm{~b}$ \\
\hline Significância & ** & ** & $n s$ & $* *$ & $n s$ & $* *$ & $* *$ & ** \\
\hline
\end{tabular}

${ }^{1} \mathrm{AM}=$ Adubação mineral; $\mathrm{VC}=$ Vermicomposto; $\mathrm{VC}+\mathrm{SB}=$ Vermicomposto + sangue bovino; RITC= Resíduo da indústria de transformação de sucos cítricos.

**e significativo por $P<0,01$

Médias seguidas pela mesma letra na coluna não diferem do nível $P<0,05$ (SNK test).

TABELA 5 - Média de massa fresca, seca no primeiro e no segundo anos, percentagem de massa seca e área das folhas ao fim do experimento, FAEM/UFPEL.

\begin{tabular}{|c|c|c|c|c|c|c|c|c|}
\hline \multirow[t]{2}{*}{ Tratamento } & \multicolumn{2}{|c|}{$\begin{array}{c}\text { Massa fresca folha }{ }^{-1} \\
\text { mg }\end{array}$} & \multicolumn{2}{|c|}{$\begin{array}{c}\text { Massa seca folha }^{-1} \\
\text { mg }\end{array}$} & \multicolumn{2}{|c|}{$\begin{array}{c}\text { Massa seca } \\
\text { folha }^{-1} \%\end{array}$} & \multirow{2}{*}{$\begin{array}{c}\text { Área foliar } \\
\begin{array}{c}\text { planta }^{-1} \\
\mathrm{~cm}^{2}\end{array} \\
1^{\circ} \text { ano } \\
\end{array}$} & \multirow{2}{*}{$\begin{array}{c}\begin{array}{c}\text { Área folha } \\
\mathrm{cm}^{2}\end{array} \\
2^{\circ} \text { ano } \\
\end{array}$} \\
\hline & $1^{\circ}$ ano & $2^{\circ}$ ano & $1^{\circ}$ ano & $2^{\circ}$ ano & $1^{\circ}$ ano & $2^{\circ}$ ano & & \\
\hline Controle & $441 \mathrm{~b}$ & $310 \mathrm{c}$ & $163 \mathrm{~b}$ & $107 \mathrm{c}$ & $30,8 \mathrm{~b}$ & $34,4 \mathrm{~b}$ & $2275 \mathrm{~b}$ & $9,6 \mathrm{~b}$ \\
\hline $\mathrm{AM}$ & $483 \mathrm{ab}$ & $478 \mathrm{a}$ & $179 \mathrm{ab}$ & 199 a & $37,0 \mathrm{a}$ & $41,7 \mathrm{a}$ & $10151 \mathrm{a}$ & $13,0 \mathrm{a}$ \\
\hline $\mathrm{VC}$ & $491 \mathrm{ab}$ & $386 \mathrm{bc}$ & $180 \mathrm{ab}$ & $144 \mathrm{~b}$ & $36,5 \mathrm{a}$ & $37,6 \mathrm{ab}$ & $4699 \mathrm{~b}$ & $11,2 \mathrm{~b}$ \\
\hline $\mathrm{VC}+\mathrm{SB}$ & $475 \mathrm{ab}$ & $455 \mathrm{ab}$ & $182 \mathrm{ab}$ & $189 \mathrm{a}$ & $38,3 \mathrm{a}$ & $41,5 \mathrm{a}$ & $11025 \mathrm{a}$ & $12,9 \mathrm{a}$ \\
\hline RIC & $558 \mathrm{a}$ & $344 \mathrm{c}$ & $202 \mathrm{a}$ & $123 \mathrm{bc}$ & $36,2 \mathrm{a}$ & $35,9 \mathrm{~b}$ & $3177 \mathrm{~b}$ & $10,5 \mathrm{~b}$ \\
\hline Significâncic & * & ** & * & ** & * & *** & $* *$ & *** \\
\hline
\end{tabular}

${ }^{1} \mathrm{AM}=$ Adubação mineral; $\mathrm{VC}=$ Vermicomposto; $\mathrm{VC}+\mathrm{SB}=$ Vermicomposto + sangue bovino; RITC= Resíduo da indústria de transformação de sucos cítricos.

** significativo por $P<0,01$

Médias seguidas pela mesma letra na coluna não diferem do nível $P<0,05$ (SNK test).

TABELA 6- Média de biomassa dos diferentes órgãos das plantas no segundo ano de cultivo, FAEM/UFPEL.

\begin{tabular}{lcccccc}
\hline Tratamentos & MF do lenho & MS do lenho & $\begin{array}{c}\text { MF das raízes } \\
\text { grossas }^{-1}\end{array}$ & $\begin{array}{c}\text { MS das } \\
\text { raízes grossas }^{-1}\end{array}$ & $\begin{array}{c}\text { MF das } \\
\text { raízes finas }\end{array}$ & $\begin{array}{c}\text { MS das } \\
\text { raízes finas }\end{array}$ \\
\cline { 2 - 7 } & g planta $^{-1}$ & $\mathrm{~g} \mathrm{planta}^{-1}$ & $\mathrm{~g} \mathrm{planta}^{-1}$ & $\mathrm{~g} \mathrm{planta}^{-1}$ & $\mathrm{~g} \mathrm{planta}^{-1}$ & $\mathrm{~g} \mathrm{planta}^{-1}$ \\
\hline Controle & $134 \mathrm{c}$ & $76 \mathrm{c}$ & $119 \mathrm{~b}$ & $58 \mathrm{~b}$ & $243 \mathrm{c}$ & $62 \mathrm{c}$ \\
Mineral & $334 \mathrm{~b}$ & $199 \mathrm{~b}$ & $277 \mathrm{a}$ & $157 \mathrm{a}$ & $310 \mathrm{~b}$ & $89 \mathrm{~b}$ \\
Vermicomposto & $184 \mathrm{c}$ & $103 \mathrm{c}$ & $142 \mathrm{~b}$ & $76 \mathrm{~b}$ & $337 \mathrm{~b}$ & $91 \mathrm{~b}$ \\
Vermicomposto + sangue & $419 \mathrm{a}$ & $243 \mathrm{a}$ & $303 \mathrm{a}$ & $170 \mathrm{a}$ & $516 \mathrm{a}$ & $150 \mathrm{a}$ \\
Composto cítrico & $172 \mathrm{c}$ & $97 \mathrm{c}$ & $142 \mathrm{~b}$ & $74 \mathrm{~b}$ & $320 \mathrm{~b}$ & $79 \mathrm{~b}$ \\
\hline Significância & $* *$ & $* *$ & $* *$ & $* *$ & $* *$ & $* *$ \\
\hline
\end{tabular}

${ }^{1} \mathrm{MF}=$ massa fresca e MS = massa seca;

** significativo por $P<0,01$.

Médias seguidas pela mesma letra na coluna não diferem do nível $\mathrm{P}<0,05$ (SNK test). 
TABELA 7 - Macronutrientes minerais (\% M.S.) nas folhas de tangerineira após o primeiro e o segundo anos de cultivo, FAEM/ UFPEL.

\begin{tabular}{lccccc|ccccc}
\hline Tratamento & \multicolumn{4}{c}{ Primeiro ano } & \multicolumn{4}{c}{ Segundo ano } \\
\hline & $\boldsymbol{N}$ & $\boldsymbol{P}$ & $\boldsymbol{K}$ & $\boldsymbol{C a}$ & $\boldsymbol{M g}$ & $\boldsymbol{N}$ & $\boldsymbol{P}$ & $\boldsymbol{K}$ & $\boldsymbol{C a}$ & $\boldsymbol{M g}$ \\
\hline Controle & $1,5 \mathrm{~b}$ & $0,3 \mathrm{a}$ & $2,2 \mathrm{bc}$ & 2,83 & 0,16 & $1,4 \mathrm{~b}$ & $0,3 \mathrm{c}$ & $1,9 \mathrm{a}$ & $3,41 \mathrm{a}$ & $0,27 \mathrm{a}$ \\
Mineral & $1,5 \mathrm{~b}$ & $0,3 \mathrm{a}$ & $2,7 \mathrm{a}$ & 3,16 & 0,12 & $1,5 \mathrm{a}$ & $0,2 \mathrm{c}$ & $0,9 \mathrm{~d}$ & $2,63 \mathrm{~b}$ & $0,17 \mathrm{~b}$ \\
Vermicomposto & $1,6 \mathrm{ab}$ & $0,3 \mathrm{a}$ & $2,6 \mathrm{a}$ & 2,6 & 0,21 & $1,1 \mathrm{c}$ & $0,5 \mathrm{~b}$ & $1,2 \mathrm{c}$ & $3,15 \mathrm{a}$ & $0,19 \mathrm{~b}$ \\
Vermicomposto + & $1,7 \mathrm{a}$ & $0,2 \mathrm{~b}$ & $1,9 \mathrm{c}$ & 2,58 & 0,13 & $1,6 \mathrm{a}$ & $0,8 \mathrm{a}$ & $1,6 \mathrm{~b}$ & $3,77 \mathrm{a}$ & $0,27 \mathrm{a}$ \\
sangue & & & & & & & & & & \\
Composto cítrico & $1,7 \mathrm{a}$ & $0,3 \mathrm{a}$ & $2,4 \mathrm{ab}$ & 2,56 & 0,19 & $1,6 \mathrm{a}$ & $0,4 \mathrm{~b}$ & $0,6 \mathrm{~d}$ & $3,61 \mathrm{a}$ & $0,26 \mathrm{a}$ \\
\hline Significância & $*$ & $*$ & $*$ & $n s$ & $n s$ & $* * *$ & $* *$ & $* *$ & $* *$ \\
\hline
\end{tabular}

$n s, * e * *$ não-significativo, significativo por $P<0,05, P<0,01$, respectivamente.

Médias seguidas pela mesma letra na coluna não diferem do nível $P<0,05$ (SNK test).

TABELA 8- Valores médios de nitrogênio total ( $\mathrm{N}$ total), nitrogênio microbiano ( $\mathrm{N}$ mic), carbono orgânico (C org), carbono microbiano (C mic) e respiração basal do solo após 2 anos de cultivo, FAEM/UFPEL.

\begin{tabular}{|c|c|c|c|c|c|}
\hline \multirow[t]{2}{*}{ Tratamentos } & $\mathrm{N}$ total & $\mathrm{N}$ mic & $\mathrm{C}$ org & $\mathrm{C}$ mic & Respiração Basal \\
\hline & -----.\%----- & ---- $\mathrm{g} \mathrm{g}^{-1}---$ & -----\% \%------ & $----\mathrm{g} \mathrm{g}^{-1}----$ & $\mathrm{mg} \mathrm{CO}_{2}^{-1} 100 \mathrm{~g}^{-1}$ solo \\
\hline Controle & $0,101 \mathrm{~b}$ & 17,9 & $0,97 \mathrm{ab}$ & $75,0 \mathrm{ab}$ & $11,0 \mathrm{~b}$ \\
\hline Mineral & $0,103 \mathrm{~b}$ & 30,1 & $0,87 \mathrm{~b}$ & $110,1 \mathrm{ab}$ & $9,9 \mathrm{~b}$ \\
\hline Vermicomposto & $0,108 \mathrm{ab}$ & 10,9 & $0,96 a b$ & $86,2 \mathrm{ab}$ & $10,8 \mathrm{~b}$ \\
\hline Vermicomposto + sangue & $0,112 \mathrm{a}$ & 15,2 & $1,08 \mathrm{a}$ & $132,2 \mathrm{a}$ & $16,3 \mathrm{a}$ \\
\hline Composto cítrico & $0,103 \mathrm{~b}$ & 14,5 & $1,04 \mathrm{a}$ & $64,8 \mathrm{~b}$ & $11,2 \mathrm{~b}$ \\
\hline Significância & $* *$ & $n s$ & $* *$ & $*$ & ** \\
\hline
\end{tabular}

$n s$, * $e^{* *}$ : não-significativo, significativo por $P<0,05$ e $P<0,01$, respectivamente.

Médias seguidas pela mesma letra na coluna não diferem do nível $\mathrm{P}<0,05$ (SNK test).

\section{CONCLUSÕES}

1-A combinação de vermicomposto + sangue, como fonte de nitrogênio, pode ser utilizada como fonte alternativa de nitrogênio para a nutrição de pomares de tanjerineira cv Clemenules sob o porta-enxerto Poncirus trifoliata, na fase inicial de desenvolvimento, em substituição à adubação mineral;

2-O vermicomposto + sangue altera de forma positiva a respiração basal e o nitrogênio total no solo.

\section{REFERÊNCIAS}

ALMEIDA, T. R. P.; LEONEL, S.; TECCHIO, M. A.; MISCHAN, M. M. Formação do pomar de tangerineira 'Poncã', em função da adubação química e orgânica. Revista Brasileira de Fruticultura, Jaboticabal, v. 27, n.2, p. 288-291, 2005.

BALDI, E.; TOSELLI, M.; MARCOLINI, G.; MARANGONI, B. Effect of mineral and organic fertilization on soil chemical, biological and physical fertility in a commercial peach orchard. Acta Horticulturae, The Hague, v.1, n. 721, p. 55-62, 2006.
CALABRESE, F. Gli agrumi: nutrizione e concimazione. Palermo: Ed. Italkali, 1988. p. 91.

COMISSÃO DE FERTILIDADE DO SOLO-RS/SC. Recomendações de adubação e calagem para os Estados do Rio Grande do Sul e Santa Catarina. Passo Fundo: Sociedade Brasileira de Ciências do Solo. Núcleo Regional do Sul, 1994. $224 p$.

DAHAMA, A.K. Organic farming for sustainable agriculture. Bikaner: Agrobios, 2002 . p. 302.

FAO, 2006. Disponível em: http://faostat.fao.org/site/339/ default.aspx

FERREIRA, A.S.; CAMARGO, F.A.O.; VIDOR, C. Utilização de microondas na avaliação da biomassa microbiana do solo. Revista Brasileira de Ciências do Solo, Viçosa, v. 23, p. 991-996, 1999.

FRENCH, N.M. Organic growers supporters get pep talk at National Conference. Citrus \& Vegetable Magazine, Lincolnshire, v. 53, n. 10, p. 32-38, 1990. 
GIUSQUINIANI, P.L.; PAGLIAI, M.; GIGLIOTTI, G.; BUSINELLI, D.; BENETTI, A. Urban waste compost: effects on physical, chemical and biochemical soil properties. Journal of Environmental Quality, Madison, n. 24, p. 175-182, 1995.

HUCHCHE, A.D.; LADANIYA, M.S.; RAM, L.; KOHLI, R.R.; SRIVASTAVA, A.K. Effect of nitrogenous fertilizers on yield, quality and shelf life of Nagpur mandarin. Indian Journal of Horticulture, New Delhi, n. 55, p. 108-112, 1998.

JONES, J. B.; BENJAMIN, W. JR.; MILLS, H. A. Plant analysis handbook. Athens: Micro-macro Publishing, 1991. p. 213.

JÖRGENSEN, R.G.; MEYER, B.; RODEN, A.; WITTKE, B. Microbial activity and biomass in mixture treatments of soil and biogenic municipal refuse compost. Biology and Fertility of Soils, Berlin, n.23, p. 43-49, 1996.

MALAVOLTA, E.; LIMA FILHO, O.F.; PICCIN, C.R.; CASALE, H. A adubação dos citros no Brasil - $\mathrm{O}$ estado da arte. In: SEMINÁRIO INTERNACIONAL DE CITROS, NUTRIÇÃO E ADUBAÇÃO, 4., 1996. Bebedouro . Anais... Bebedouro-SP : Fundação Cargill 1996. p.1-14.

MAZZANTI, F. Acquisizione di nutrienti da fertilizzanti organici e minerali e stato nutrizionale del melo. 2002. Thesis $(\mathrm{PhD})$ - Dipartimento di Colture Arboree, Alma Mater Studiorum, Università di Bologna, Bologna , 2002.

NIKLASCH, H.; JÖRGENSEN, R.G. Decomposition of peat, biogenic municipal waste compost, and shrub/grass compost added in different rayes to a silt loam. Journal of Plant Nutrition and Soil Science, Berlin, n. 14, p. 54-60, 2001.

PARODA, R.S. Doil fertility management certain issues. Journal of the Indian Society of Soil Science, New Delhi, n. 47, p. 580$581,1999$.

PEDUCCI, P. Enzyme activity and microbial biomass in a field soil amended with municipal refuse. Biology and Fertility of Soils, Berlin, n. 14, p. 54-60, 1992.
QIANGSHENG, W.; RENXUE, X.; ZHENGJIA, H. Effect of Arbuscular Mycorrhiza on the Drought Tolerance of Poncirus trifoliata Seedlings. Frontiers of Forestry in China, Beijing, v. 1, n. 1, p. 100-104, 2006.

SCHUBERT, A.; BODRINO, C.; GRIBAUDO, I. Vesciculararbuscular mycorrhizal inoculation of kiwifruit (Actinidia chinensis) micropropagated plants. Agronomie, Paris, n. 12, p. 847-850, 1992.

SCUDELLARI, D.; TAGLIAVINI, M.; MARANGONI, B.; RUBBI, L.; CAPPUCCI, V.; PELLICONI, F. Azoto nel frutteto, una concimazione calibrata. Terra e Vita, Bologna, n. 29, p. 29-32, 1998.

SORRENTI, G. La gestione idrico-nutrizionale nella melicoltura moderna. Phytomagazine, Rivoli Veronese,, v. 52, n. 6. p. 53-64, 2006.

SRIVISTAVA, A.K.; SINGH, S.; MARATHE, R.A. Journal of Sustainable Agriculture, Binghamton, NY, v.19, n. 3, p. 5-29, 2002.

STEVENSON, F.J. Cycles of soil: carbon, nitrogen, phosphorus, sulfur, micronutrients. New York: John Wiley \& Sons, 1986. 380.

STOTZKY, G. Microbial respiration. In: BLACK, C.A., (Ed.). Methods of soil analysis. Madison: American Society of Agronomy, 1965. v.2, p. 1551-1572.

TEDESCO, M.J.; GIANELLO, C.; BISSANI, C.A. Análises de solos, plantas e outros materiais. 2. ed. Porto Alegre: Universidade Federal do Rio Grande do Sul, 1995. 174p. (Boletim Técnico, 5).

TOSELLI, M.; BALDI, E.; MARCOLINI, G.; MARANGONI, B. La concimazione di pre-impianto delle colture arboree da frutto. L'informatore Agrario, Verona, n. 47, p. 51-54, 2004.

VANCE, E.D.; BROOKES, P.C.; JENKINSON, D.S. An extraction method for measuring soil microbial biomass C. Soil Biology \& Biochemistry, Netherlands, v. 19, n. 6, p. 703-707, 1987. 